\title{
The missing Wendland functions
}

\author{
Robert Schaback
}

Received: 13 November 2008 / Accepted: 12 September 2009 /

Published online: 24 September 2009

(C) The Author(s) 2009. This article is published with open access at Springerlink.com

\begin{abstract}
The Wendland radial basis functions (Wendland, Adv Comput Math 4:389-396, 1995) are piecewise polynomial compactly supported reproducing kernels in Hilbert spaces which are norm-equivalent to Sobolev spaces. But they only cover the Sobolev spaces

$$
H^{d / 2+k+1 / 2}\left(\mathbf{R}^{d}\right), k \in \mathbf{N}
$$

and leave out the integer order spaces in even dimensions. We derive the missing Wendland functions working for half-integer $k$ and even dimensions, reproducing integer-order Sobolev spaces in even dimensions, but they turn out to have two additional non-polynomial terms: a logarithm and a square root. To give these functions a solid mathematical foundation, a generalized version of the "dimension walk" is applied. While the classical dimension walk proceeds in steps of two space dimensions taking single derivatives, the new one proceeds in steps of single dimensions and uses "halved" derivatives of fractional calculus.
\end{abstract}

Keywords Sobolev spaces - Compactly supported radial basis functions • Kernels $\cdot$ Hypergeometric functions $\cdot$ Positive definite functions • Fractional calculus

Mathematics Subject Classifications (2000) 33C90 • 41A05 • 41A15 • 41A30 • $41 \mathrm{~A} 63 \cdot 65 \mathrm{D} 07 \cdot 65 \mathrm{D} 10$

Communicated by Joe Ward.

R. Schaback $(\bowtie)$

Institut für Numerische und Angewandte Mathematik, Universität Göttingen,

Lotzestrasse 16-18, 37083 Göttingen, Germany

e-mail: schaback@math.uni-goettingen.de 


\section{Introduction}

In a wide range of applications from Machine Learning to Meshless Methods [11], positive definite kernels have proven to be very useful tools. They arise naturally as reproducing kernels of Hilbert spaces of continuous functions, and they often come in translation- and rotation-invariant form as radial basis functions

$$
K(x, y)=\phi\left(\|x-y\|_{2}\right) \text { for all } x, y \in \mathbf{R}^{d}
$$

on $\mathbf{R}^{d}$ with a smooth univariate function $\phi:[0, \infty) \rightarrow \mathbf{R}$. Typical examples are furnished by the Gaussian $\phi(r):=\exp \left(-r^{2}\right)$ or the Sobolev/Matérn functions $\phi(r)=K_{v}(r) r^{\nu}$. The latter have special importance in geostatistics [8], and are the reproducing kernels of Sobolev spaces $H^{m}\left(\mathbf{R}^{d}\right)$ for $v=m-d / 2>0$. They involve the $K_{v}$ Bessel functions of third kind and decay exponentially towards infinity.

Unfortunately, these kernels are not compactly supported, but by arguments using convolutions of compactly supported functions, it is quite clear that there must be compactly supported positive definite radial basis functions of arbitrary prescribed smoothness. The quest for explicit formulas for such functions started with "Euclid's hat" in 1993 [4, 9] and continued with piecewise polynomial radial basis functions of $\mathrm{Wu}$ [16] and Wendland [14] in 1995. While Euclid's hat is not differentiable and Wu's functions have zeros in their Fourier transform, Wendland's functions $\phi_{d, k}$ have no such drawbacks. They are polynomials on $[0,1]$ and yield positive definite $C^{2 k}$ radial basis functions on $\mathbf{R}^{d}$. Given these properties, their polynomial degree $\lfloor d / 2\rfloor+3 k+1$ is minimal, and they are reproducing kernels of Hilbert spaces isomorphic to Sobolev space $H^{d / 2+k+1 / 2}\left(\mathbf{R}^{d}\right)$.

But since this means that integer-order Sobolev spaces in even dimensions are not covered, this paper produces the "missing" functions by allowing half-integer values of $k$. They still are compactly supported, but they are polynomials with additional logarithmic and square-root terms. For example, the function

$$
\phi_{2,1 / 2}(r)=\frac{\sqrt{2}}{3 \sqrt{\pi}}\left(3 r^{2} \log \left(\frac{r}{1+\sqrt{1-r^{2}}}\right)+\left(2 r^{2}+1\right) \sqrt{1-r^{2}}\right)
$$

on $[0,1]$ and extended by zero to $[0, \infty)$ yields a positive definite radial basis function on $\mathbf{R}^{2}$ and it will turn out to be the reproducing kernel in an isomorphic copy of to $H^{2}\left(\mathbf{R}^{2}\right)$. See Fig. 1 for a plot of this function.

The paper will first review the standard construction process for Wendland's functions by the "dimension walk". It usually steps through space dimensions in steps of two while taking successive derivatives. To reach the missing intermediate space dimensions, the dimension walk has to proceed in steps of single dimensions. But then it has to use "half-derivatives" from what is called "fractional calculus" [7]. This generates the functions we need, and we 
Fig. 1 The missing Wendland function $\phi_{2,1 / 2}$

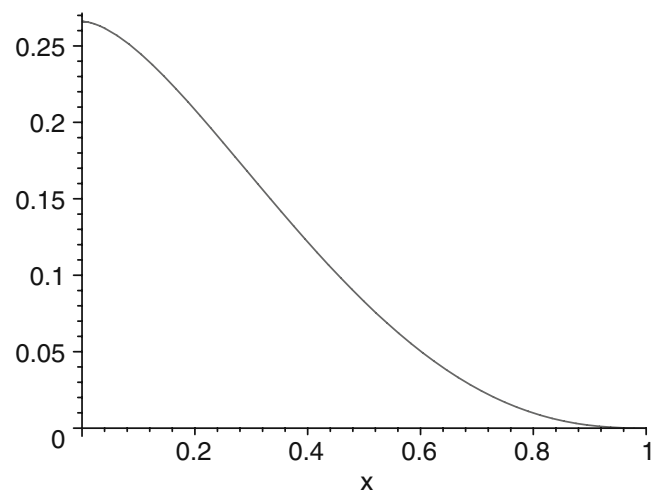

provide a MAPLE program for explicit calculation. Finally, we add proofs for the required properties of the new functions and close with an outlook that connects everything to hypergeometric functions.

\section{Radial transforms}

To start with, we repeat part of the machinery of fractional calculus from [12] and [10]. Proof details can be found there. It is well-known that a radial basis function

$$
\Phi(x):=\phi\left(\|x\|_{2}\right), x \in \mathbf{R}^{d}
$$

has a radial $d$-variate Fourier transform

$$
\hat{\Phi}(\omega)=\|\omega\|_{2}^{-(d-2) / 2} \int_{0}^{\infty} \phi(r) r^{d / 2} J_{(d-2) / 2}\left(r\|\omega\|_{2}\right) d r
$$

if the integral exists. It allows the Fourier transform of a radial function to be written as a univariate Hankel transform.

We now introduce $t=r^{2} / 2$ as a new variable, writing a radial basis function $\phi$ as

$$
\phi\left(\|\cdot\|_{2}\right)=f\left(\|\cdot\|_{2}^{2} / 2\right) .
$$

At first sight, this " $f$-form" seems somewhat strange. Indeed, the $f$-form of the familiar Wendland function $\phi_{3,1}(r)=(1-r)_{+}^{4}(4 r+1)$ will be the nonpolynomial function $f_{3,1}(t)=(1-\sqrt{2 t})_{+}^{4}(4 \sqrt{2 t}+1)$. But the following mathematical analysis of radial functions in $f$-form turns out to be much simpler than in "normal" form, and this fact should be familiar from Schoenberg's connection of positive definite radial basis functions to completely monotone functions [13]. 
Now (3) for $v=(d-2) / 2$, when written in $f$-form on both sides, turns into

$$
\left(F_{v} f\right)(s):=\int_{0}^{\infty} f(t) t^{v} H_{v}(t s) d t
$$

with the function

$$
\left(\frac{z}{2}\right)^{-v} J_{v}(z)=: H_{v}\left(z^{2} / 4\right)=\sum_{k=0}^{\infty} \frac{\left(-z^{2} / 4\right)^{k}}{k ! \Gamma(k+v+1)}
$$

for $z \in \mathbf{C}$ and $v \in \mathbf{C}$ with $\Re v>0$. Note that (5) is a generalization of the Fourier transform on radial functions, allowing Fourier transforms for spaces of fractal dimension, because $v=(d-2) / 2$ need not be a half-integer. The functions

$$
\left(\frac{z}{2}\right)^{-v} J_{v}(z)=H_{v}\left(z^{2} / 4\right)
$$

are called oscillatory radial basis functions by Fornberg et al. [3]. The above derivation shows that they are of central importance, because they supply the generalized Fourier transforms of general radial functions. We shall use them frequently in what follows.

Using derivative formulae for the $H_{v}$ functions obtainable from those of the $J_{v}$ functions, one gets

$$
-\frac{d}{d s} F_{v}(f)(s)=F_{v+1}(f)(s) \text { and }\left(F_{v+1}\left(-f^{\prime}\right)\right)(s)=\left(F_{v}(f)\right)(s) .
$$

Going back to $v=(d-2) / 2$, these are the basic features of the dimension walk, but we shall need them later in steps of dimension one:

Theorem 1 If the mentioned Fourier transforms and derivatives exist,

- the $(d+2)$-variate Fourier transform of a radial function after the f-form substitution (4) is the negative univariate derivative of the d-variate Fourier transform in f-form, and

- the d-variate Fourier transform of a radial function in $f$-form is the $(d+2)$ variate Fourier transform of the negative derivative of $f$.

Note that all continuous and compactly supported $f$ will have smooth generalized Fourier transforms $F_{v}(f)$ in all dimensions, making the left-hand part of (7) valid for all dimensions. However, the application of the right-hand part of (7) is restricted by the smoothness of $f$.

The dimension walk, expressed via derivatives, is extremely useful when programming with radial basis functions. It turns out that all the relevant classes of radial basis functions, when written in $f$ form, are invariant under differentiation and integration, while the $F_{v}$ operators map the class to another one which is also closed under differentiation and integration. Implementing the general class in $f$ form automatically yields an implementation of all derivatives. 
But we shall need fractional derivatives to generalize all of this. To this end, [12] introduces a scale of integral operators

$$
I_{\alpha}(f)(t):=\int_{t}^{\infty} f(s) \frac{(s-t)^{\alpha-1}}{\Gamma(\alpha)} d s
$$

for all $\alpha>0, t \geq 0$, defined on continuous functions on $[0, \infty)$ with compact support or exponential decay at infinity. As the authors of [12] found out later, these are closely connected to the Weyl derivative operators of fractional calculus and were introduced also in [6] for use in the dimension walk. The simplest special case is

$$
I_{1}(f)(t):=\int_{t}^{\infty} f(s) d s
$$

with the inverse

$$
I_{-1}(f)(r):=-f^{\prime}(r)
$$

we already had above, doing the dimension walk. These operators satisfy

$$
I_{\alpha} \circ I_{\beta}=I_{\alpha+\beta}
$$

for all $\alpha, \beta>0$. Thus the "semi-integration" operator

$$
I_{1 / 2}(f)(t)=\int_{t}^{\infty} \frac{f(s)}{\sqrt{\pi(s-t)}} d s
$$

satisfies $I_{1 / 2} \circ I_{1 / 2}=I_{1}$. These definitions can be extended to let

$$
I_{\alpha} \circ I_{\beta}=I_{\alpha+\beta}
$$

hold for all $\alpha, \beta \in \mathbf{R}$ and on suitable domains, but we refer to [10, 12] for details. Note that all of these operators are intended to work on $f$-forms of radial functions, not on their normal form. They are closely connected but not identical to the standard operators of fractional calculus.

With these operators at hand, [10, 12] generalize the dimension walk to

$$
\begin{aligned}
\left(F_{\mu} \circ F_{\nu}\right)(f) & =I_{\nu-\mu}(f) \\
F_{\mu} & =I_{\nu-\mu} F_{\nu} \\
F_{\nu} & =F_{\mu} I_{\nu-\mu}
\end{aligned}
$$

as far as the operators are applicable, in particular for $v \geq \mu$ and on compactly supported smooth functions, and this is what we need for generating the missing Wendland functions.

\section{Application to Wendland functions}

Due to a result of Askey [1] the radial truncated power functions

$$
A_{\mu}(\cdot):=\left(1-\|\cdot\|_{2}\right)_{+}^{\mu}
$$


are positive definite on $\mathbf{R}^{d}$ for integer $\mu \geq\lfloor d / 2\rfloor+1$, because they have a strictly positive radial Fourier transform in these cases. Formally, we allow $\mu$ to be positive and real from now on and turn to positive definiteness later.

The $f$-form of Askey functions is

$$
a_{\mu}(s):=(1-\sqrt{2 s})_{+}^{\mu} .
$$

Since the $I_{\alpha}$ operators preserve compact supports and are applicable to $a_{\mu}$ for all $\alpha, \mu>0$, we can define

$$
a_{\mu, \alpha}:=I_{\alpha}\left(a_{\mu}\right) \text { with } a_{\mu, 0}=a_{\mu} .
$$

Going back to "normal" form, the functions

$$
\left.\psi_{\mu, \alpha}(r):=\left(I_{\alpha}\left(a_{\mu}\right)\right)\left(r^{2} / 2\right)\right)
$$

are well-defined and supported in $[0,1]$ for all $\alpha, \mu>0$ and can be called general Wendland functions. At this point, we do not know for which parameters they are positive definite in which space dimension. They can be represented as

$$
\begin{aligned}
\psi_{\mu, \alpha}(x) & =\int_{0}^{\infty}(1-\sqrt{2 s})_{+}^{\mu} \frac{\left(s-x^{2} / 2\right)_{+}^{\alpha-1}}{\Gamma(\alpha)} d s \\
& =\int_{x}^{1} t(1-t)^{\mu} \frac{\left(t^{2}-x^{2}\right)^{\alpha-1}}{\Gamma(\alpha) 2^{\alpha-1}} d t
\end{aligned}
$$

for $x \in[0,1]$. This coincides with the formula (9.2.20) in [10] (see also Gneiting [5]) and it is normally used only for integer $\alpha, \mu$ to get the polynomial representations of the Wendland functions directly. The connection to the $\phi_{d, k}$ notation in Wendland's monograph [15] to the above formula is via

$$
\phi_{d, k}=\psi_{\lfloor d / 2\rfloor+k+1, k},
$$

because there are good reasons to pick the smallest known $\mu$ which guarantees $\psi_{\mu, k}$ to be positive definite in $d$ dimensions for a given $d$, and this minimal $\mu$ is $\lfloor d / 2\rfloor+k+1$ for integer $k$. The case of half-integer $\alpha$ or $\mu$ of the formula (11) was not treated so far, though it clearly generates functions with support in $[0,1]$.

\section{Construction algorithm}

If $\mu$ and $\alpha$ are integers, the resulting function in (11) is a single polynomial of degree $\mu+2 \alpha$ in the variable $x=\|\cdot\|_{2}$ on its support, and the integral can be 
directly calculated. However, one can try to calculate the integral in general, and this is partially done by the MAPLE snippet

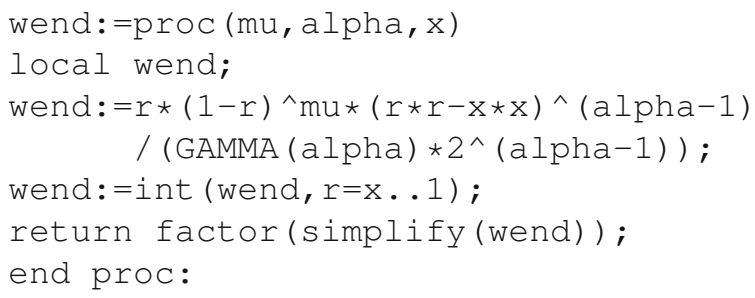

which runs for all reasonable and fixed choices of $\mu$ and $\alpha$ where one halfinteger is allowed, while it fails if both $\mu$ and $\alpha$ are genuine half-integers. Since $\psi_{d / 2+\alpha+1 / 2, \alpha}=\phi_{d, \alpha}$ will be proven in Corollary 1 below to be reproducing in spaces norm-equivalent to $H^{d / 2+\alpha+1 / 2}\left(\mathbf{R}^{d}\right)$ for half-integer $\alpha$ and even $d$, the above snippet generates the missing Wendland functions for half-integer $\alpha$ and integer $\mu$. The first interesting case is $\mu=d=2, \alpha=1 / 2$ leading to (2) plotted in Fig. 1. It is a reproducing kernel in an isomorphic copy of $H^{2}\left(\mathbf{R}^{2}\right)$. Using the abbreviations

$$
\begin{aligned}
& L(x):=\log \left(\frac{x}{1+\sqrt{1-x^{2}}}\right) \\
& S(x):=\sqrt{1-x^{2}}
\end{aligned}
$$

the next cases are

$$
\begin{aligned}
\psi_{2,3 / 2}(x)= & \frac{-\sqrt{2}}{60 \sqrt{\pi}}\left(15 x^{4} L(x)+\left(8 x^{4}+9 x^{2}-2\right) S(x)\right) \\
\psi_{2,5 / 2}(x)= & \frac{\sqrt{2}}{2520 \sqrt{\pi}}\left(105 x^{6} L(x)+\left(48 x^{6}+87 x^{4}-38 x^{2}+8\right) S(x)\right) . \\
\psi_{4,1 / 2}(x)= & \frac{\sqrt{2}}{30 \sqrt{\pi}}\left(\left(45 x^{4}+60 x^{2}\right) L(x)+\left(16 x^{4}+83 x^{2}+6\right) S(x)\right), \\
\psi_{4,3 / 2}(x)= & \frac{-\sqrt{2}}{420 \sqrt{\pi}}\left(\left(105 x^{6}+210 x^{4}\right) L(x)\right. \\
& \left.+\left(32 x^{6}+247 x^{4}+40 x^{2}-4\right) S(x)\right) \\
\psi_{4,5 / 2}(x)= & \frac{\sqrt{2}}{30240 \sqrt{\pi}}\left(\left(945 x^{8}+2520 x^{6}\right) L(x)\right. \\
& \left.+\left(256 x^{8}+263 x^{6}+690 x^{4}-136 x^{2}+16\right) S(x)\right) \\
& \frac{\sqrt{2}}{280 \sqrt{\pi}}\left(\left(525 x^{6}+2100 x^{4}+840 x^{2}\right) L(x)\right. \\
& \left.+\left(128 x^{6}+1779 x^{4}+1518 x^{2}+40\right) S(x)\right) . \\
\psi_{6,1 / 2}(x) &
\end{aligned}
$$


The general case will be proven below to be of the form

$$
\psi_{2 m,(2 \ell-1) / 2}(x)=x^{2 \ell} p_{m, \ell}\left(x^{2}\right) L(x)+q_{m, \ell}\left(x^{2}\right) S(x)
$$

with polynomials $p_{m, \ell}$ of degree $m-1$ and $q_{m, \ell}$ of degree $m-1+\ell$. These functions do not seem to be directly available via the technique of Buhmann [2].

\section{Theoretical analysis}

We now provide rigid proofs of the statements made above. Recall that the standard Askey functions satisfy $F_{\nu}\left(a_{\mu}\right)>0$ iff $\mu \geq\lfloor v\rfloor+2$ where $\mu$ is an integer and $v=(d-2) / 2$ may be a half-integer. Furthermore, we know that $F_{v}(f)>0$ and $d=2 v+2 \in \mathbf{N}$ imply that $f\left(\|\cdot\|_{2}^{2} / 2\right)$ is positive definite on $\mathbf{R}^{d}=\mathbf{R}^{2 v+2}$.

Theorem 2 For all $\alpha \in \mathbf{N} / 2$ and all $\mu \in \mathbf{N}$ with

$$
\mu \geq\lfloor d / 2+\alpha\rfloor+1
$$

the generalized Wendland function $\psi_{\mu, \alpha}$ is positive definite on $\mathbf{R}^{d}$.

Proof We use the identity $F_{v+\alpha}=F_{\nu} \circ I_{\alpha}$ from (9) for $a_{\mu}$ and get

$$
F_{v+\alpha} a_{\mu}=F_{v}\left(I_{\alpha}\left(a_{\mu}\right)\right)
$$

which is valid for all $\alpha, \mu>0$ and all $v>-1 / 2$. But we restrict ourselves to the case

$$
v+\alpha \in \mathbf{Z} / 2, v+\alpha \geq-1 / 2,
$$

and apply Askey's result for $d=2 v+2 \alpha+2$ to get that the left-hand side is strictly positive whenever

$$
\mu \geq\lfloor v+\alpha\rfloor+2
$$

Looking at the right-hand side of (16) and introducing a new dimension with $(d-2) / 2=v$, we see that the function $I_{\alpha}\left(A_{\mu}\right)$ is positive definite on $\mathbf{R}^{d}$ if (15) holds.

Theorem 3 For $\alpha \in \mathbf{N} / 2$, the d-variate Fourier transform $\mathscr{F}_{d}\left(\psi_{\mu, \alpha}\right)$ of $\psi_{\mu, \alpha}$ with

$$
\mu=\lfloor d / 2+\alpha\rfloor+1 \geq 3
$$

satisfies

$$
\mathscr{F}_{d}\left(\psi_{\mu, \alpha}\right)(r)=\Theta\left(r^{-(d+2 \alpha+1)}\right) \text { for } r \rightarrow \infty
$$


Proof Using (16) and the transition to the $f$-form, we get

$$
\begin{aligned}
\mathscr{F}_{d} \psi_{\mu, \alpha}(r) & =F_{\frac{d-2}{2}} a_{\mu, \alpha}\left(r^{2} / 2\right) \\
& =F_{\frac{d-2}{2}+\alpha} a_{\mu, 0}\left(r^{2} / 2\right) \\
& =\mathscr{F}_{d+2 \alpha} a_{\mu}\left(r^{2} / 2\right) \\
& =\mathscr{F}_{d+2 \alpha} A_{\mu}(r)
\end{aligned}
$$

to see that the $d$-variate Fourier transform of $\psi_{\mu, \alpha}$ in normal form is identical to the $(d+2 \alpha)$-variate Fourier transform of the Askey function $A_{\mu}$ in normal form.

Since (17) allows two possible connections between $\mu$ and $d$, we fix $d$ and $\alpha$ first, define $d^{\prime}:=d+2 \alpha$ and look at the two possibilities $d^{\prime}=2 \mu-1$ and $d^{\prime}=2 \mu-2$ which share $\left\lfloor d^{\prime} / 2\right\rfloor=\lfloor d / 2+\alpha\rfloor=\mu-1$. From section 10.5 of [15] we cite

$$
\begin{aligned}
& \left(\mathscr{F}_{2 \mu-1} A_{\mu}\right)(r)=\Theta\left(r^{-2 \mu}\right) \\
& \left(\mathscr{F}_{2 \mu-2} A_{\mu}\right)(r)=\Theta\left(r^{-2 \mu+1}\right)
\end{aligned}
$$

for integer $\mu \geq 3$. Then (19) yields the assertion.

Note that the above argument excludes $\mu=2, \alpha=1 / 2$ and $d=1$, thus not proving that $\psi_{2,1 / 2}$ is reproducing in a space equivalent to $H^{3 / 2}\left(\mathbf{R}^{1}\right)$. But it generalizes (1) for half-integers $\alpha$ and even-dimensional spaces, since $\mu \geq 3$ holds in such cases:

Corollary 1 For integers $m \geq 1$ and $n \geq 0$, the generalized Wendland function $\psi_{m+n+1, n+1 / 2}$ taken for even dimensions $d=2 m$ is reproducing in a Hilbert space which is isomorphic to Sobolev space $H^{m+n+1}\left(\mathbf{R}^{2 m}\right)=H^{d / 2+\alpha+1 / 2}\left(\mathbf{R}^{d}\right)$ where $\alpha=n+1 / 2$.

\section{Inductive construction}

This chapter proves the representation (14) for the missing Wendland functions $\psi_{2 m,(2 \ell-1) / 2}$ for all $\ell$ and $m$. We start with $\ell=1$ and general $m$.

Theorem 4 The general Wendland functions $a_{2 m, 1 / 2}(t)$ have the form

$$
\begin{aligned}
a_{2 m, 1 / 2}(t) & :=\int_{t}^{1 / 2} \frac{(1-\sqrt{2 s})^{2 m}}{\sqrt{\pi(s-t)}} d s \\
& =P_{m, 0}(t) L(\sqrt{2 t})+Q_{m, 0}(t) S(\sqrt{2 t})
\end{aligned}
$$

with polynomials $P_{m, 0}, Q_{m, 0}$ of degree $m$ and

$$
\begin{aligned}
P_{m, 0}(1 / 2) & =Q_{m, 0}(1 / 2) \\
P_{m, 0}(0) & =0
\end{aligned}
$$

for all $m \geq 1$. 
Proof We can also consider

$$
\begin{aligned}
a_{2 m, 1 / 2}\left(x^{2} / 2\right) & =\frac{\sqrt{2}}{\sqrt{\pi}} \int_{x}^{1} \frac{r(1-r)^{2 m}}{\sqrt{r^{2}-x^{2}}} d r \\
& =P_{m, 0}\left(x^{2} / 2\right) L(x)+Q_{m, 0}\left(x^{2} / 2\right) S(x)
\end{aligned}
$$

and transform this by $z:=\sqrt{r^{2}-x^{2}}$ into

$$
a_{2 m, 1 / 2}\left(x^{2} / 2\right)=\frac{\sqrt{2}}{\sqrt{\pi}} \int_{0}^{\sqrt{1-x^{2}}}\left(1-\sqrt{x^{2}+z^{2}}\right)^{2 m} d z .
$$

Applying the binomial formula leads to terms

$$
g_{k}(x):=\int_{0}^{\sqrt{1-x^{2}}}\left(x^{2}+z^{2}\right)^{k} d z
$$

for all $k \in \mathbf{Z} / 2$ with $0 \leq k \leq m$ combining into

$$
a_{2 m, 1 / 2}\left(x^{2} / 2\right)=\frac{\sqrt{2}}{\sqrt{\pi}} \sum_{j=0}^{2 m}(-1)^{j}\left(\begin{array}{c}
2 m \\
j
\end{array}\right) g_{j / 2}(x) .
$$

We get

$$
\begin{aligned}
g_{k+1}(x) & =\int_{0}^{\sqrt{1-x^{2}}}\left(x^{2}+z^{2}\right)\left(x^{2}+z^{2}\right)^{k} d z \\
& =x^{2} g_{k}(x)+\int_{0}^{\sqrt{1-x^{2}}} z^{2}\left(x^{2}+z^{2}\right)^{k} d z \\
& =x^{2} g_{k}(x)+\frac{\sqrt{1-x^{2}}}{2(k+1)}-\frac{1}{2(k+1)} \int_{0}^{\sqrt{1-x^{2}}}\left(x^{2}+z^{2}\right)^{k+1} d z \\
& =x^{2} g_{k}(x)+\frac{\sqrt{1-x^{2}}}{2(k+1)}-\frac{1}{2(k+1)} g_{k+1}(x)
\end{aligned}
$$

such that

$$
\begin{aligned}
g_{k+1}(x)\left(1+\frac{1}{2(k+1)}\right) & =x^{2} g_{k}(x)+\frac{\sqrt{1-x^{2}}}{2(k+1)} \\
g_{k+1}(x) & =\frac{2 k+2}{2 k+3} x^{2} g_{k}(x)+\frac{S(x)}{2 k+3}
\end{aligned}
$$

is a useful recursion that boils everything down to

$$
\begin{aligned}
g_{0}(x) & =\sqrt{1-x^{2}} \\
g_{1 / 2}(x) & =-\frac{1}{2} x^{2} L(x)+\frac{\sqrt{1-x^{2}}}{2}=\frac{1}{2}\left(S(x)-x^{2} L(x)\right) .
\end{aligned}
$$

This gives polynomials $p_{j}, q_{j}, r_{j}$ of degree at most $j$ with

$$
\begin{aligned}
g_{j}(x) & =S(x) p_{j}\left(x^{2}\right) \\
g_{j-1 / 2}(x) & =L(x) q_{j}\left(x^{2}\right)+S(x) r_{j-1}\left(x^{2}\right)
\end{aligned}
$$


such that

$$
\begin{aligned}
\frac{\sqrt{\pi}}{\sqrt{2}} a_{2 m, 1 / 2}\left(x^{2} / 2\right)= & \sum_{i=0}^{m}\left(\begin{array}{c}
2 m \\
2 i
\end{array}\right) g_{i}(x) \\
& -\sum_{i=1}^{m}\left(\begin{array}{c}
2 m \\
2 i-1
\end{array}\right) g_{i-1 / 2}(x) \\
= & S(x)\left(\sum_{i=0}^{m}\left(\begin{array}{c}
2 m \\
2 i
\end{array}\right) p_{i}\left(x^{2}\right)-\sum_{i=1}^{m}\left(\begin{array}{c}
2 m \\
2 i-1
\end{array}\right) r_{i-1}\left(x^{2}\right)\right) \\
& -L(x) \sum_{i=1}^{m}\left(\begin{array}{c}
2 m \\
2 i-1
\end{array}\right) q_{i}\left(x^{2}\right)
\end{aligned}
$$

is of the required form.

We have to check the additional conditions (21). Since $q_{j}$ has no constant term, we get $P_{m, 0}(0)=0$. To prove the conditions at $1 / 2$, we remark that evaluation of an $f$ form at $1 / 2$ means evaluation of the standard form at 1 . We rewrite the representation (22) in terms of $z=\sqrt{1-x^{2}}$ as

$$
\begin{aligned}
a_{2 m, 1 / 2}\left(\left(1-z^{2}\right) / 2\right)= & P_{m, 0}\left(\left(1-z^{2}\right) / 2\right) L\left(\sqrt{1-z^{2}}\right) \\
+ & Q_{m, 0}\left(\left(1-z^{2}\right) / 2\right) S\left(\sqrt{1-z^{2}}\right)
\end{aligned}
$$

and now evaluation at $x=1$ means evaluation at $z=0$. We expand the terms at $z=0$ to get

$$
\begin{aligned}
L\left(\sqrt{1-z^{2}}\right) & =-z+\mathscr{O}\left(z^{3}\right) \\
S\left(\sqrt{1-z^{2}}\right) & =z
\end{aligned}
$$

to see that

$$
a_{2 m, 1 / 2}(1 / 2)=-P_{m, 0}(1 / 2)+Q_{m, 0}(1 / 2)
$$

which vanishes due to the support of the $f$ form ending at $1 / 2$.

Theorem 5 The representation

$$
a_{2 m,(2 \ell+1) / 2}(s)=P_{m, \ell}(s) L(\sqrt{2 s})+Q_{m, \ell}(s) S(\sqrt{2 s})
$$

with polynomials $P_{m, \ell}, Q_{m, \ell}$ of degree $m+\ell$ and

$$
\begin{aligned}
P_{m, \ell}(1 / 2) & =Q_{m, \ell}(1 / 2) \\
P_{m, 0}(0) & =0
\end{aligned}
$$

holds for all $m \geq 1$ and all $\ell \geq 0$.

Proof We know that (23) holds for $\ell=0$ and all $m \geq 1$, and thus we proceed by induction on $\ell$. By the standard dimension walk rules (9) we have to construct $P_{m, \ell}, Q_{m, \ell}$ from $P_{m, \ell-1}, Q_{m, \ell-1}$ to satisfy

$$
-a_{2 m, 2 \ell-1}(s)=a_{2 m,(2 \ell+1) / 2}(s)^{\prime} .
$$


The induction recipe will be to define $P_{m, \ell}$ by

$$
\begin{aligned}
P_{m, \ell}(s)^{\prime} & =-P_{m, \ell-1}(s) \\
P_{m, \ell}(0) & =0
\end{aligned}
$$

and to define $Q_{m, \ell}$ via

$$
Q_{m, \ell}(s)^{\prime}(1-2 s)-Q_{m, \ell}(s)=-\frac{P_{m, \ell}(s)}{2 s}-Q_{m, \ell-1}(s)(1-2 s) .
$$

It can easily be shown that the above equation is uniquely solvable for $Q_{m, \ell}$ of degree $m+\ell$, and it implies

$$
Q_{m, \ell}(1 / 2)=P_{m, \ell}(1 / 2) .
$$

Now we have to evaluate both sides of (24) in order to finish the induction. We need the derivatives

$$
\begin{aligned}
L^{\prime}(x) & =\frac{1}{x \sqrt{1-x^{2}}} \\
S^{\prime}(x) & =\frac{-x}{\sqrt{1-x^{2}}} \\
L(\sqrt{2 s})^{\prime} & =\frac{1}{2 s \sqrt{1-2 s}} \\
S(\sqrt{2 s})^{\prime} & =\frac{-1}{\sqrt{1-2 s}}
\end{aligned}
$$

and get

$$
\begin{aligned}
a_{2 m,(2 \ell+1) / 2}(s)^{\prime}= & P_{m, \ell}(s)^{\prime} L(\sqrt{2 s})+P_{m, \ell}(s) L(\sqrt{2 s})^{\prime} \\
& +Q_{m, \ell}(s)^{\prime} S(\sqrt{2 s})+Q_{m, \ell}(s) S(\sqrt{2 s})^{\prime} \\
= & P_{m, \ell}(s)^{\prime} L(\sqrt{2 s})+P_{m, \ell}(s) \frac{1}{2 s \sqrt{1-2 s}} \\
& +Q_{m, \ell}(s)^{\prime} S(\sqrt{2 s})-Q_{m, \ell}(s) \frac{1}{\sqrt{1-2 s}} .
\end{aligned}
$$

Focusing on the log terms above and in $a_{2 m,(2 \ell-1) / 2}(s)$, we see that they are correct due to our choice of $P_{m, \ell}$. Now we are left to prove that

$$
-Q_{m, \ell-1}(s) S(\sqrt{2 s})=-Q_{m, \ell-1}(s) \sqrt{1-2 s}
$$

coincides with

$$
P_{m, \ell}(s) \frac{1}{2 s \sqrt{1-2 s}}+Q_{m, \ell}(s)^{\prime} \sqrt{1-2 s}-Q_{m, \ell}(s) \frac{1}{\sqrt{1-2 s}} .
$$

But the latter is identical to

$$
\frac{P_{m, \ell}(s)+2 s(1-2 s) Q_{m, \ell}(s)^{\prime}-2 s Q_{m, \ell}(s)}{2 s \sqrt{1-2 s}} .
$$


Introducing $z:=\sqrt{1-2 s}$ we have to prove

$$
\begin{aligned}
-Q_{m, \ell-1}\left(\frac{1-z^{2}}{2}\right) z= & \frac{1}{z\left(1-z^{2}\right)}\left(P_{m, \ell}\left(\frac{1-z^{2}}{2}\right)\right. \\
& +Q_{m, \ell}^{\prime}\left(\frac{1-z^{2}}{2}\right) z^{2}\left(1-z^{2}\right)-Q_{m, \ell}\left(\frac{1-z^{2}}{2}\right)\left(1-z^{2}\right) .
\end{aligned}
$$

But since our construction yields

$$
\begin{aligned}
P_{m, \ell}(1 / 2) & =Q_{m, \ell}(1 / 2) \\
P_{m, \ell}(0) & =0,
\end{aligned}
$$

the critical denominator cancels, and our definition of $Q_{m, \ell}$ does the job.

Note that Theorems 4 and 5 imply the representation (14). The special form of the $p_{m, \ell}$ part is due to the fact that (25) does not change the number of monomial terms, which is fixed at startup in Theorem 4, but only blows the degree up by one.

\section{Open problems}

Readers will have noticed that we did not deal with the two remaining cases of generalized Wendland functions $\psi_{\mu, k}$ : those with integer $k$ and half-integer $\mu$ and those with both indices being half-integer. We did not focus on these because they are less promising. This is based on some hypotheses, confirmed for special cases, which we now formulate.

First, there is quite some evidence that

$$
F_{\nu}\left(a_{\mu, 0}\right)(t)=\Theta\left(t^{-v-3 / 2}\right) \text { for } t \rightarrow \infty
$$

holds in full generality, in particular independent of $\mu$, and is positive for all

$$
\mu \geq\left\lceil v+\frac{3}{2}\right\rceil
$$

not only in the special cases related to (20). Note here that the standard case is recovered by

$$
\lceil v+3 / 2\rceil=\lceil d / 2+1 / 2\rceil=\lfloor d / 2\rfloor+1 \text { if } v=(d-2) / 2,
$$

but one could allow more general $\nu$. The above assertions should follow from a very thorough inspection of chapters 6 and 10 of [15], and they generalize Theorems 2 and 3. Since large $\mu$ do not pay off, Wendland's notation (12) based on the smallest $\mu$ yielding positive definiteness for dimension $d$ makes a lot of sense.

If the above is assumed, the minimal $\mu$ for $\psi_{\mu, \alpha}$ to be positive definite in generalized dimension $d$ is

$$
\mu=\lceil\alpha+d / 2+1 / 2\rceil .
$$


Then (26) can be applied for $v=\alpha+(d-2) / 2$, proving that the generalized Wendland function $\psi_{\lceil\alpha+d / 2+1 / 2\rceil, \alpha}$ is reproducing in Hilbert spaces isomorphic to $H^{m}\left(\mathbf{R}^{d}\right)$ for $m=\alpha+d / 2+1 / 2$. This should be expected for all real $d$ and $\alpha$, leading to compactly supported reproducing kernels also in fractional-order Sobolev spaces.

To generate the integer-order Hilbert spaces in all dimensions, it therefore suffices to use the classical Wendland functions and those we described here. The $\mu$ parameter is not of central importance. However, fractional Sobolev spaces will require fractional $\alpha$, but our integral operators (8) will allow to generate these either directly via (10) and (11), if the integral can be calculated, or from a polynomial Wendland function via

$$
I_{\alpha} a_{\mu}=I_{\alpha-\lfloor\alpha\rfloor} I_{\lfloor\alpha\rfloor} a_{\mu}=I_{\alpha-\lfloor\alpha\rfloor} a_{\mu,\lfloor\alpha\rfloor}
$$

if the operator $I_{\alpha-\lfloor\alpha\rfloor}$ can be explicitly evaluated on monomials.

The case of $\psi_{\mu, \alpha}$ with half-integer $\mu \in(\mathbf{N} / 2) \backslash \mathbf{N}$ and integer $\alpha$ can easily be handled with the methods of Section 4 and the MAPLE program presented there. It generates polynomials times $\sqrt{1-x}$. But under the above assertions, genuinely half-integer $\mu$ do not seem to be minimally chosen. Things would be different if there was half-integer leeway in the condition (27), but $F_{1 / 2} a_{7 / 4,0}$ is not positive, thanks to MAPLE.

Finally we remark that there are good chances to put all of this into a uniform theory based on hypergeometric functions. But we leave these things open.

Acknowledgement Special thanks go to a referee for careful reading and useful suggestions.

Open Access This article is distributed under the terms of the Creative Commons Attribution Noncommercial License which permits any noncommercial use, distribution, and reproduction in any medium, provided the original author(s) and source are credited.

\section{References}

1. Askey, R.: Radial characteristic functions. Technical Report 1262, Univ. of Wisconsin, 1973. MRC Technical Sum. Report.

2. Buhmann, M.D.: Radial functions on compact support. Proc. Edinb. Math. Soc. 41, 33-46 (1998)

3. Fornberg, B., Larsson, E., Wright, G.: A new class of oscillatory radial basis functions. Comput. Math. Appl. 51(8):1209-1222, 2006.

4. Gneiting, T.: Radial positive definite functions generated by Euclid's hat. J. Multivar. Anal. 69(1), 88-119 (1999)

5. Gneiting, T.: Compactly supported correlation functions. J. Multivar. Anal. 83(2), 493-508 (2002)

6. Matheron, G.: Les Variables régionaliseés Et Leur Estimation. Masson, Paris (1965)

7. Oldham, K.B., Spanier, J.: The Fractional Calculus; Theory and Applications of Differentiation and Integration to Arbitrary Order. Academic, London (1974)

8. Pardo-Iguzquiza, E., Chica-Olmoa, M.: Geostatistics with the matern semivariogram model: a library of computer programs for inference, kriging and simulations. Comput. Geosci. 34, 1073-1079 (2008) 
9. Schaback, R.: Creating surfaces from scattered data using radial basis functions. In: Lyche, T., Daehlen, M., Schumaker, L.L. (eds.) Mathematical Methods for Curves and Surfaces, pp. 477496. Vanderbilt University Press, Nashville (1995)

10. Schaback, R.: Reconstruction of multivariate functions from scattered data. Manuscript. http://www.num.math.uni-goettingen.de/schaback/research/group.html (1997)

11. Schaback, R., Wendland, H.: Kernel techniques: from machine learning to meshless methods. Acta Numer. 15, 543-639 (2006)

12. Schaback, R, Wu, Z.: Operators on radial basis functions. J. Compt. Appl. Math. 73, 257-270 (1996)

13. Schoenberg, I.J.: Metric spaces and completely monotone functions. Annal. Math. 39, 811-841 (1938)

14. Wendland, H.: Piecewise polynomial, positive definite and compactly supported radial functions of minimal degree. Adv. Comput. Math. 4, 389-396 (1995)

15. Wendland, H.: Scattered Data Approximation. Cambridge Monographs on Applied and Computational Mathematics. Cambridge University Press, Cambridge (2005)

16. Wu, Z.: Compactly supported positive definite radial functions. Adv. Comput. Math. 4, 283$292(1995)$ 